\title{
Relative skeletal muscle mass and non-alcoholic fatty liver disease: from association to causation
}

\author{
Hee Yeon Kim ${ }^{1}$, Jong Young Choi ${ }^{1}$, Yong-Moon Park ${ }^{2}$ \\ ${ }^{1}$ Division of Hepatology, Department of Internal Medicine, College of Medicine, The Catholic University of Korea, Seoul, Korea; ${ }^{2}$ Epidemiology \\ Branch, National Institute of Environmental Health Sciences, National Institutes of Health, Research Triangle Park, NC, USA \\ Correspondence to: Yong-Moon Park, MD, MS, PhD. National Institute of Environmental Health Sciences, Mail Drop A3-53, 111 T.W. Alexander \\ Drive, Research Triangle Park, NC 27709, USA. Email: mark.park@nih.gov. \\ Comment on: Kim G, Lee SE, Lee YB, et al. Relationship between relative skeletal muscle mass and nonalcoholic fatty liver disease: a 7-year \\ longitudinal study. Hepatology 2018;68:1755-68.
}

Submitted Mar 04, 2019. Accepted for publication Apr 16, 2019.

doi: 10.21037/hbsn.2019.04.13

View this article at: http://dx.doi.org/10.21037/hbsn.2019.04.13

The prevalence of nonalcoholic fatty liver disease (NAFLD) and its associated metabolic risk factors, including obesity and metabolic syndrome, have increased over the last decade in both developed and developing countries (1). NAFLD is considered to be the hepatic manifestation of metabolic syndrome and insulin resistance which is one of multiple hits leading to NAFLD (2). Because skeletal muscle is an important endocrine organ that responds to the insulin, loss of skeletal muscle mass decreases glucose utilization and induces insulin resistance, which in turn may contribute to the development of NAFLD (3).

Sarcopenia, defined as a decrease in skeletal muscle mass, and NAFLD share similar underlying mechanisms and risk factors, including insulin resistance, chronic inflammation, vitamin $\mathrm{D}$ deficiency, and physical inactivity (3). In line with these shared characteristics, it has been reported that low skeletal muscle mass is associated with an elevated risk of NAFLD. This relationship between sarcopenia and NAFLD was also independent of other well-known metabolic risk factors (4-6). However, the cross-sectional nature of these studies limited the identification of causal relationships between the two.

In a paper published in the November 2018 issue of Hepatology (7), Kim et al. investigated the longitudinal relationship between relative skeletal muscle mass and NAFLD occurrence or resolution. In this single-center, large, 7-year, Korean longitudinal study, they assessed the associations of relative skeletal muscle mass and alterations in relative skeletal muscle mass over time with the occurrence or resolution of NAFLD. Among 12,624 individuals who did not have NAFLD at enrollment, $14.8 \%$ developed NAFLD, and the higher muscle mass was inversely associated with incident NAFLD [HR (highest vs. lowest tertile) $0.44,95 \% \mathrm{CI}, 0.38-0.51$ ]. Interestingly, NAFLD resolved in 79 subjects (2.7\%) among 2,943 participants with initial NAFLD and the higher muscle mass was positively associated with the resolution of baseline NAFLD [HR (highest $v s$. lowest tertile): 2.09, 95\% CI, 1.02-4.28].

In line with previous cross-sectional studies, this study by Kim et al. (7) confirmed the association between sarcopenia and NAFLD, suggesting a potential causal link. Recognizing the skeletal muscle as a myokine-secreting endocrine organ, which can influence other metabolic tissues such as the liver, may guide to understand its possible causative function in developing NAFLD (8). In addition, myostatin is a potent factor for inhibiting skeletal muscle. Hepatic stellate cells also have Activin IIbr, a receptor for myostatin (9). This finding poses a doubt on whether fatty liver leads to skeletal muscle loss by activating myostatin in the skeletal muscle, or whether low skeletal muscle mass induces hepatic damage by activating hepatic stellate cells. It is challenging to identify whether sarcopenia is a cause or a consequence of fatty liver, or both.

It is worth noting that in the study by Kim et al. (7), a rise in muscle mass over a year showed significant favorable impacts either on the occurrence of NAFLD or the resolution of baseline NAFLD, even after adjusting 
for initial skeletal muscle index (SMI) and glycometabolic risk factors. Currently, Food and Drug Administration (FDA)-approved therapies are not available for NAFLD, and lifestyle modification, including diet and exercise, are recommend in the first line management of NAFLD. In terms of the role of nutrition, excessive consumption of sucrose or fructose may cause hepatic steatosis (10), and alter body composition and muscle protein pool (11). Moreover, lower vitamin D concentrations were shown to be associated with sarcopenia as well as NAFLD (3). Considering the lack of effective therapeutic drugs, nutrition intervention can serve as one of the principal strategies for prevention and management of NAFLD in the context of liver-muscle interactions. The mechanisms contributing to the reduction in hepatic fat after physical exercise in NAFLD are attributed to alterations in energy balance and insulin sensitivity (12). The beneficial role of skeletal muscle mass on the development or resolution of NAFLD is one of the promising mechanisms which might explain the favorable effect of exercise in reducing liver fat.

NAFLD comprises a broad spectrum of disease ranging from simple steatosis to nonalcoholic steatohepatitis (NASH) (13). The risk of progression to end-stage liver disease is higher in patients with steatohepatitis or significant fibrosis than in patients with simple steatosis. It is important to point out that in Kim's study (7), authors defined NAFLD using hepatic steatosis index (HSI), one of the noninvasive prediction model for fatty liver. Although HSI is one of the noninvasive scoring systems for NAFLD with high areas under the curve in Korean individuals, the HSI scoring system is not the gold standard method to diagnose NAFLD, and it also cannot discriminate steatohepatitis from simple steatosis (13). It has been demonstrated that sarcopenia was related with NASH and significant liver fibrosis $(4,6,14)$. Longitudinal studies investigating the influences of relative skeletal muscle mass and changes in the composition of muscle mass over time on steatohepatitis or fibrosis are urgently warranted.

The European Working Group on Sarcopenia in Older People recently suggested the definition of sarcopenia as progressive decline in both skeletal muscle mass and muscle function (15). In the study by Kim et al. (7), muscle quality, such as muscle power and muscle fiber types, were not considered in diagnosing sarcopenia. Although the study by Kim and coworkers (7) has limitations, including the use of a noninvasive scoring system for simple steatosis and the use of muscular indices, it is novel in that not only baseline SMI, but also an increase in SMI over time was advantageous for either preventing incident NAFLD or improving existing NAFLD.

In conclusion, both relative skeletal muscle mass at baseline and increases in muscle mass over time have a beneficial effect on both developing NAFLD and improving baseline NAFLD. These results imply that preventing sarcopenia may be a preventive and therapeutic intervention for the management of NAFLD. Further prospective interventional studies that endeavor to investigate changes in skeletal muscle mass and NAFLD are warranted.

\section{Acknowledgments}

This research was supported in part by the Intramural Program in the NIH, National Institute of Environmental Health Sciences.

\section{Footnote}

Conflicts of Interest: The authors have no conflicts of interest to declare.

\section{References}

1. Seto WK, Yuen MF. Nonalcoholic fatty liver disease in Asia: emerging perspectives. J Gastroenterol 2017;52:164-74.

2. Alam S, Mustafa G, Alam M, et al. Insulin resistance in development and progression of nonalcoholic fatty liver disease. World J Gastrointest Pathophysiol 2016;7:211-7.

3. Zhai Y, Xiao Q. The Common Mechanisms of Sarcopenia and NAFLD. Biomed Res Int 2017;2017:6297651.

4. Kim HY, Kim CW, Park CH, et al. Low skeletal muscle mass is associated with non-alcoholic fatty liver disease in Korean adults: the Fifth Korea National Health and Nutrition Examination Survey. Hepatobiliary Pancreat Dis Int 2016;15:39-47.

5. Lee YH, Jung KS, Kim SU, et al. Sarcopaenia is associated with NAFLD independently of obesity and insulin resistance: Nationwide surveys (KNHANES 2008-2011). J Hepatol 2015;63:486-93.

6. Moon JS, Yoon JS, Won KC, et al. The role of skeletal muscle in development of nonalcoholic Fatty liver disease. Diabetes Metab J 2013;37:278-85.

7. Kim G, Lee SE, Lee YB, et al. Relationship Between Relative Skeletal Muscle Mass and Nonalcoholic Fatty Liver Disease: A 7-Year Longitudinal Study. Hepatology 2018;68:1755-68. 
8. Pedersen BK, Febbraio MA. Muscles, exercise and obesity: skeletal muscle as a secretory organ. Nat Rev Endocrinol 2012;8:457-65.

9. Bhanji RA, Narayanan P, Allen AM, et al. Sarcopenia in hiding: The risk and consequence of underestimating muscle dysfunction in nonalcoholic steatohepatitis. Hepatology 2017;66:2055-65.

10. Ouyang X, Cirillo P, Sautin Y, et al. Fructose consumption as a risk factor for non-alcoholic fatty liver disease. J Hepatol 2008;48:993-9.

11. Gatineau E, Savary-Auzeloux I, Migne C, et al. Chronic Intake of Sucrose Accelerates Sarcopenia in Older Male Rats through Alterations in Insulin Sensitivity and Muscle Protein Synthesis. J Nutr 2015;145:923-30.

Cite this article as: Kim HY, Choi JY, Park YM. Relative skeletal muscle mass and non-alcoholic fatty liver disease: from association to causation. HepatoBiliary Surg Nutr 2019;8(5):509511. doi: 10.21037/hbsn.2019.04.13
12. Romero-Gómez M, Zelber-Sagi S, Trenell M. Treatment of NAFLD with diet, physical activity and exercise. J Hepatol 2017;67:829-46.

13. Oh H, Jun DW, Saeed WK, et al. Non-alcoholic fatty liver diseases: update on the challenge of diagnosis and treatment. Clin Mol Hepatol 2016;22:327-35.

14. Petta S, Ciminnisi S, Di Marco V, et al. Sarcopenia is associated with severe liver fibrosis in patients with nonalcoholic fatty liver disease. Aliment Pharmacol Ther 2017;45:510-8.

15. Cruz-Jentoft AJ, Baeyens JP, Bauer JM, et al. Sarcopenia: European consensus on definition and diagnosis: Report of the European Working Group on Sarcopenia in Older People. Age Ageing 2010;39:412-23. 